\section{Intravitreal aflibercept for macular oedema secondary to central retinal vein occlusion in patients with prior treatment with bevacizumab or ranibizumab}

\begin{abstract}
Purpose To report the visual and anatomic outcomes in eyes with macular oedema (MO) secondary to central retinal vein occlusion (CRVO) that were switched from either intravitreal bevacizumab or ranibizumab to intravitreal aflibercept.

Methods Two-center retrospective chart review. Eyes with MO secondary to CRVO that received a minimum of three intravitreal injections of bevacizumab or ranibizumab and were switched to intravitreal aflibercept for persistent or recurrent MO not responding to either bevacizumab and/or ranibizumab. Results In all 42 eyes of 42 patients were included in the study. The median visual acuity before the switch was 20/126, 1 month after the first injection of aflibercept 20/89 $(P=0.0191)$, and at the end of the follow-up 20/100 $(P=0.2724)$. The median CRT before the switch was $536 \mu \mathrm{m}, 1$ month after the first injection of aflibercept $293.5 \mu \mathrm{m}(P=0.0038)$, and at the end of the follow-up $279 \mu \mathrm{m}$ $(P=0.0013$ compared to before the switch).

The median number of weeks between injections before the switch was 5.6 and after the switch was $7.6(P<0.0001)$.

Conclusion Converting eyes with refractory MO due to CRVO to aflibercept can result in stabilization of the vision, improved macular anatomy, and extension of the injection interval. Eye (2016) 30, 79-84; doi:10.1038/eye.2015.175; published online 9 October 2015
\end{abstract}

\section{Introduction}

Recent advances in the pharmacologic treatment of macular oedema (MO) from central retinal
TD Papakostas ${ }^{1}$, L Lim¹ ${ }^{1}$ T van Zyl ${ }^{1}$, JB Miller ${ }^{1}$, BS Modjtahedi ${ }^{1}$, CM Andreoli ${ }^{2}, \mathrm{D}$ Wu${ }^{1}$, LH Young ${ }^{1}$, IK Kim ${ }^{1}$, DG Vavvas ${ }^{1}$, DD Esmaili ${ }^{3}$, D Husain ${ }^{1}$, D Eliott $^{1}$ and LA Kim ${ }^{1}$

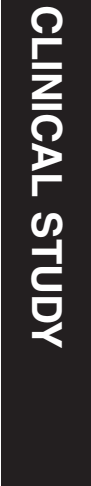

${ }^{1}$ Retina Service,

Massachusetts Eye and Ear Infirmary, Harvard Medical School, Boston, MA, USA

vein occlusion (CRVO) has resulted in a dramatic improvement in visual prognosis. Therapeutic options were historically limited, as the Central Retinal Vein Occlusion Study (CVOS) failed to demonstrate the benefit of macular grid laser for MO. ${ }^{1}$ During the last 5 years, new treatments have demonstrated efficacy and safety in several multicenter randomized controlled clinical trials. These treatments include intravitreally delivered corticosteroids, as shown in the SCORE trial ${ }^{2}$ and the GENEVA trial, ${ }^{3}$ and intravitreal injections of agents against vascular endothelial growth factor (VEGF). Ranibizumab, a humanized, affinity-matured antibody fragment against all isoforms of VEGF-A, has been shown in the CRUISE trial ${ }^{4}$ to provide rapid improvement in visual acuity (VA) and MO after CRVO. Studies have also demonstrated positive results with the use of bevacizumab, 5,6 the parent molecule of ranibizumab, which has been used off-label as a cost-effective alternative to ranibizumab. Anti-VEGF agents are associated with a more favorable safety profile compared with intravitreal steroids with respect to intraocular pressure and cataract, and thus are first-line treatment for MO due to CRVO by most retina specialists.

The eyes enrolled in the CRUISE study experienced significant improvement in their VA with an average gain of 14.9 letters at 6 months ${ }^{4}$ and 13.9 letters at 12 months. ${ }^{7}$ However, $23.1 \%$ of eyes had persistent oedema (defined as central retinal thickness (CRT) greater than $250 \mu \mathrm{m}$ ) at 6 months $^{4}$ and $22.3 \%$ at 12 months. ${ }^{7}$ Bhisitkul et $a l^{8}$ reported that eyes that responded early to treatment with ranibizumab in the CRUISE
${ }^{2}$ Harvard Vanguard Medical Associates, Boston, MA, USA

${ }^{3}$ Retina-Vitreous Associates Medical Group, Los Angeles, CA, USA

Correspondence: D Eliott, Associate Director, Retina Service, Massachusetts Eye and Ear Infirmary. Stelios Evangelos Gragoudas Associate Professor of Ophthalmology, Harvard Medical School, Boston, MA 02114, USA Tel: +1 617573 3736; Fax: +1 6175733698 E-mail: dean_eliott@ meei.harvard.edu or LA Kim, Instructor in Ophthalmology, Massachusetts Eye and Ear Infirmary, Harvard Medical School, Boston, MA 02114, USA

Tel: +1 6173915896 .

E-mail: leo_kim@

meei.harvard.edu

Received: 20 May 2015 Accepted in revised form: 12 July 2015 Published online: 9 October 2015

This work was presented at the 2015 ARVO meeting in Denver, Colorado, USA and the 2015 ASRS meeting in Vienna, Austria 
study (CRT at month 3 of $250 \mu \mathrm{m}$ or less) tended to have better visual gains over 6 and 12 months compared with eyes that exhibited a late or incomplete response.

More recently, aflibercept, a decoy fusion receptor protein comprising key domains of human VEGF receptors 1 and 2 with immunoglobulin- $\mathrm{F} \mathrm{Fc}^{9}$ has been added to our armamentarium for eyes with $\mathrm{MO}$ after CRVO. It binds all isoforms of VEGF-A, VEGF-B, and placental growth factor (PIGF), and its efficacy and safety have been established in the GALILEO ${ }^{10}$ and COPERNICUS ${ }^{11}$ trials.

Aflibercept was approved by the FDA for use in CRVO with MO in 2012. Since then, retina specialists have been offering it as a treatment option in patients with $\mathrm{MO}$ as first-line treatment or in those who did not respond to treatment with ranibizumab or bevacizumab. ${ }^{12}$ The purpose of this study was to examine the visual and anatomic outcomes in eyes with $\mathrm{CRVO}$ and $\mathrm{MO}$ that was refractory to treatment with ranibizumab or bevacizumab and were switched to aflibercept.

\section{Materials and methods}

This is a retrospective, interventional, non-comparative case series from an academic retina practice (Massachusetts Eye and Ear Infirmary) and a private practice (Harvard Vanguard Medical Associates). All the patients received treatment at the Retina Service of Massachusetts Eye and Ear Infirmary (DW, LHY, IKK, DGV, DDE, DH, DE, and LAK) and at the Harvard Vanguard Medical Associates (CMA). The study protocol was approved by the Institutional Review Board of Massachusetts Eye and Ear Infirmary (IRB protocol

\#14-135H) and Harvard Vanguard Medical Associates (IRB protocol \# 696993-1). The study adheres to the Declarations of Helsinki and all Massachusetts and US laws.

Eyes that were included in the study had prior treatment for MO secondary to CRVO with a minimum of three intravitreal injections of either bevacizumab $(1.25 \mathrm{mg} / 0.05 \mathrm{ml})$ or ranibizumab $(0.5 \mathrm{mg} / 0.05 \mathrm{ml})$ and then switched to intravitreal aflibercept $(2 \mathrm{mg} / 0.05 \mathrm{ml})$. The eyes were switched to aflibercept for persistent $\mathrm{MO}$ or if they had recurrent oedema that initially resolved after treatment with bevacizumab and/or ranibizumab and later on did not respond to repeated injections of the same agents. The identification of eligible eyes was done through billing records at Massachusetts Eye and Ear Infirmary and Harvard Vanguard Medical Associates with the ICD-9 code for CRVO as the search criterion (362.35) for patients seen from September 2012 (date aflibercept received FDA approval for MO in CRVO) up to September 2014.

Treatment schedules, injection intervals, and injection techniques were at the discretion of each retina specialist.
The parameters measured at each visit were nonstandardized Snellen VA and CRT with optical coherence tomography (OCT). The VA before the switch was the acuity measured the day that the patient had the first injection of aflibercept. All Snellen VA values were converted to $\log M A R$ for statistical analysis. The CRT was measured from the thickness map of a spectral domain OCT platform. The two platforms utilized were the Cirrus OCT by Zeiss (Carl Zeiss Meditec, Dublin, CA, USA) and the Spectralis OCT by Heidelberg (Heidelberg Engineering, Heidelberg, Germany). Patients were imaged with the same imaging modality on every followup visit. Regarding the injection interval data, the mean injection interval before and after the switch was calculated for each patient and these values were used for the subsequent statistical analysis.

Statistical analysis was performed with the Prism software by GraphPad (La Jolla, CA, USA). All values were checked for normal distribution with the D'Agostino and Pearson omnibus normality test. Median values with range are reported in data distributed in a non-Gaussian manner, and mean values with 1 standard deviation are reported in normally distributed datasets. The Wilcoxon paired signed ranked test was used for the comparisons of injection intervals. VA data were analyzed with the Friedman test and the Dunn's post hoc test to correct for multiple comparisons. OCT data were analyzed with the Kruskal-Wallis test and the Dunn's post hoc test to correct for multiple comparisons. Categorical data were analyzed with Fisher's exact test. Missing values were not imputed. The level of statistical significance was set at 0.05 .

Table 1 Demographics, baseline, and follow-up characteristics

\begin{tabular}{lc}
\hline Demographics & \\
$\quad$ Patients (eyes) & $42(42)$ \\
Mean age, years (+ - 1 SD) & $69.8(+/-13.8)$ \\
Women, $n(\%)$ & $16(38)$ \\
Diabetes, $n(\%)$ & $14(33)$ \\
$\quad$ Diabetic retinopathy, $n(\%)$ & $6(14)$ \\
$\begin{array}{l}\text { Duration of symptoms in months, median } \\
\text { (range) }\end{array}$ & $1.25(0.23-12)$ \\
Previous steroid injections, ${ }^{*} n(\%)$ & $3(7)$ \\
Scatter panretinal photocoagulation, $n(\%)$ & $10(24)$ \\
$\quad$ Capillary non-perfusion, $n(\%)$ & $3(7)$ \\
$\quad$ Iris and/or retinal NV, $n$ (\%) & $7(17)$ \\
Previous injections, median (range) & $7(3-26)$ \\
Follow-up in months pre switch, median & $12(3-53.5)$ \\
(range) & \\
Aflibercept injections, mean (+/ - 1 SD) & $7.4(+/-4.7)$ \\
Follow-up in months post switch, median & $14(3-22.2)$ \\
(range) & \\
\hline
\end{tabular}

*Triamcinolone or Dexamethasone implant.

**Bevacizumab or Ranibizumab. 


\section{Results}

\section{Demographics and baseline characteristics}

Forty-two eyes of 42 patients were included in the study. Sixteen $(38 \%)$ patients were female and 26 were male $(62 \%)$. The mean age of the patients was 69.8 years (+/ - 13.8). Forty-one eyes had a CRVO and one eye had a hemiretinal vein occlusion. Fourteen (33\%) patients had diabetes and six (14\%) of them had concurrent mild nonproliferative diabetic retinopathy. The median duration of symptoms before the patients had any treatment was 1.25 months (0.23-12). The data are summarized in Table 1.

\section{Treatments}

Prior to the switch to aflibercept The median number of intravitreal injections prior to the switch to aflibercept with either bevacizumab or ranibizumab was 7 (3-26).

Sixty-six percent of the injections before the switch were bevacizumab and $34 \%$ ranibizumab. Thirty patients $(71.5 \%)$ had partial response of the MO after bevacizumab and/or ranibizumab (11 patients received only bevacizumab, 4 patients received only ranibizumab, and the remaining 15 patients received both agents, median number of injections 6.5 ), while 4 patients (9.5\%) exhibited no response at all ( 2 patients received only bevacizumab, 1 patient received only ranibizumab, and 1 patient received both agents, median number of injections 4.5). In eight patients $(19 \%)$, the MO initially resolved but later recurred and did not respond to repeated injections of the same agent (five patients received only bevacizumab, one patient received only ranibizumab, and two patients received both agents, median number of injections 11).

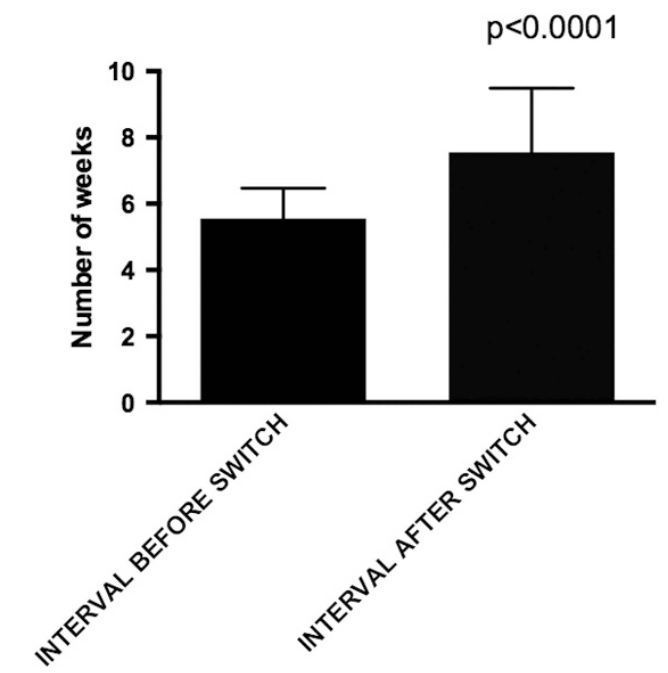

Figure 1 Injection interval before and after the switch to aflibercept. The error bars are interquartile range.
Ten eyes (24\%) had prior panretinal photocoagulation for capillary non-perfusion ( 3 eyes, $7 \%$ ) or retinal and/or iris neovascularization (7 eyes, $17 \%)$. Three eyes $(7 \%$ ) had prior intravitreal triamcinolone injection(s) or intravitreal dexamethasone implant(s) (Ozurdex, Allergan, Irvine, CA, USA). The median duration of follow-up after the first injection of bevacizumab or ranibizumab before the switch to aflibercept was 12 months (3-53.5).

After the switch to aflibercept The mean number of aflibercept injections was $7.4(+/-4.7)$. The median follow-up after the first aflibercept injection was 14 months (3-22.2).

Injection interval The median interval between injections before the switch to aflibercept was 5.6 weeks (4-18) and after the switch was 7.6 weeks $(4-23)(P<0.0001$, Figure 1). No eye after the conversion to aflibercept

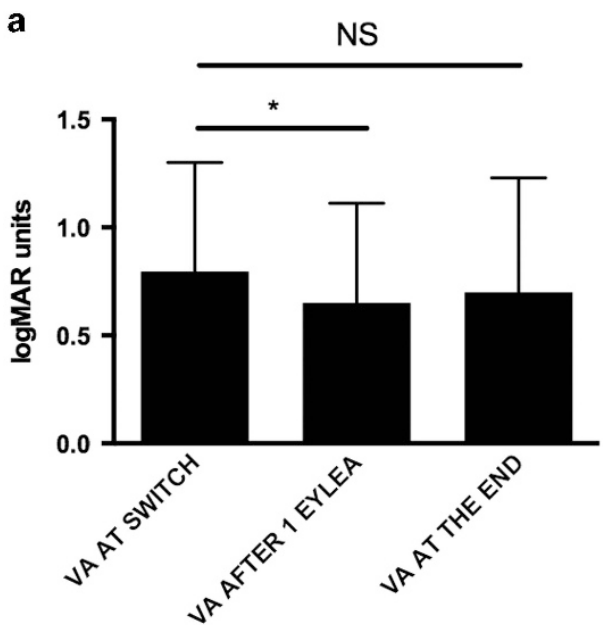

Follow-up

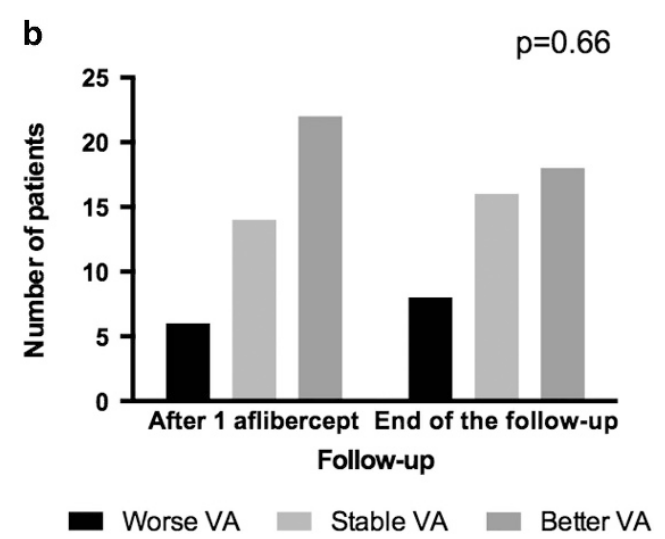

Figure 2 (a) Visual acuity before the switch, after one injection of aflibercept, and at the end of the follow-up. The error bars represent interquartile range. (b) Number of patients with worse, same, or better vision after one injection of aflibercept and at the end of the follow-up. ${ }^{*} P<0.05$. 
required more frequent injections than before the conversion.

Complications One eye had a vitreous hemorrhage 2 days after a bevacizumab injection that resolved without additional treatment. No patient had any other adverse event associated with the injection procedure or the treatment medication.

\section{Visual outcomes}

The median VA before the switch was 20/126 (logMAR 0.8 , range $0.3-1.6), 1$ month after the first injection of aflibercept 20/89 (logMAR 0.65, range 0-1.6, $P=0.0191)$ and at the end of the follow-up 20/100 (logMAR 0.84, range $0-1.6, P=0.2724$ compared with before the switch). The eyes achieved statistically significant better VA after one injection of aflibercept that was not sustained at the end of the follow-up (Figure 2a). At 1 month after the first injection of aflibercept, 6 eyes (14\%) had worse VA than before the switch, 14 eyes (33\%) had the same VA, and 22 eyes $(53 \%)$ experienced an improvement. Similar rates of acuity change were seen at the final follow-up visit. Eight eyes (19\%) had worse VA compared with before the switch, 16 eyes (38\%) remained stable, and 18 eyes (43\%) had better VA (Figure 2b, $P=0.66$ ).

\section{Anatomic outcomes}

The median CRT before the switch was $536 \mu \mathrm{m}$ (246-1003), 1 month after the first injection of aflibercept was $293.5 \mu \mathrm{m}(185-713)(P=0.0038)$, and at the end of the follow-up was $279 \mu \mathrm{m}$ (171-927) $(P=0.0013$ compared

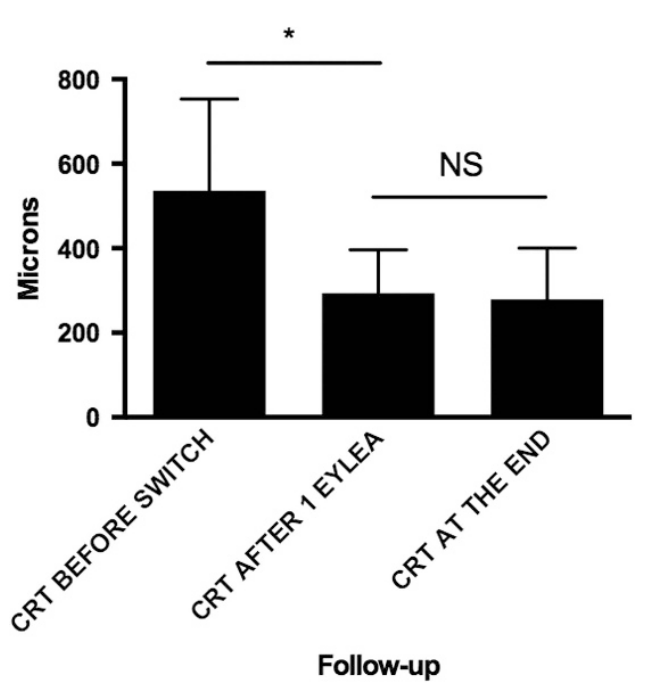

Figure 3 CRT before the switch, after one injection of aflibercept, and at the end of the follow-up. The error bars represent interquartile range. ${ }^{*} P<0.05$. with before the switch). The median thickness at the end of the follow-up was not statistically significantly different than the thickness after one injection of aflibercept $(P>0.99)$ (Figure 3). Nineteen eyes (45\%) had persistent intraretinal and/or subretinal fluid at the end of the follow-up. Fifteen of 23 (65\%) eyes without oedema at the end of the follow-up had attenuation of the ellipsoid zone on the OCT. Seven of 23 eyes (30\%) also had significant thinning of the retina (CRT less than $200 \mu \mathrm{m})$.

\section{Discussion}

Aflibercept has recently been added to our treatment options for patients with MO after CRVO. Eyes enrolled in the COPERNICUS trial ${ }^{13}$ gained 17.3 letters at 6 months after six monthly injections of aflibercept, and similar results were reported in the GALILEO study. ${ }^{14}$ These effects were maintained after additional as needed dosing at 1 year ${ }^{15}$ and 18 months, $^{16}$ respectively. Aflibercept has higher binding affinity for VEGF compared with ranibizumab or bevacizumab, ${ }^{9}$ which is an attractive feature for eyes that do not respond to treatment with ranibizumab or bevacizumab. Bhisitkul et al ${ }^{8}$ demonstrated that eyes with $\mathrm{MO}$ and CRVO can display different patterns of anatomic response to the treatment with intravitreal ranibizumab. They defined as early responders the patients with CRT $250 \mu \mathrm{m}$ or less at 3 months after the initiation of treatment in the CRUISE study. Late or non-responders had reduced visual outcomes at 6 and 12 months compared with the early responders. More specifically, the late or nonresponders had a gain of 11.9 letters at 6 months compared with 15.9 letters for the early responders and a similar trend was noted at 12 months (10.1 vs 15 letters). The importance of these findings is highlighted when considering that approximately one out of four eyes with $\mathrm{MO}$ in CRVO will have persistent oedema at 6 and 12 months based on the CRUISE study results.

In our study, the patients had on average a good anatomic response after the first injection of aflibercept, which was maintained until the end of the follow-up (Figure 4). Despite the decrease in CRT, our eyes in this study did not experience an improvement in VA, but rather a stabilization of their VA at the end of the followup. Our patients had statistically significant better VA after one injection of aflibercept, but this was not sustained until the end of the follow-up. Plausible explanations for this phenomenon include foveal damage secondary to the long-standing oedema or retinal capillary nonperfusion in the fovea. These could be true, as $65 \%$ of the patients without oedema had attenuation or loss of the ellipsoid zone in the oveal area at the end of the follow-up. In addition, chronic VEGF inhibition has been suggested to lead to retinal degeneration. ${ }^{17}$ Similar results 

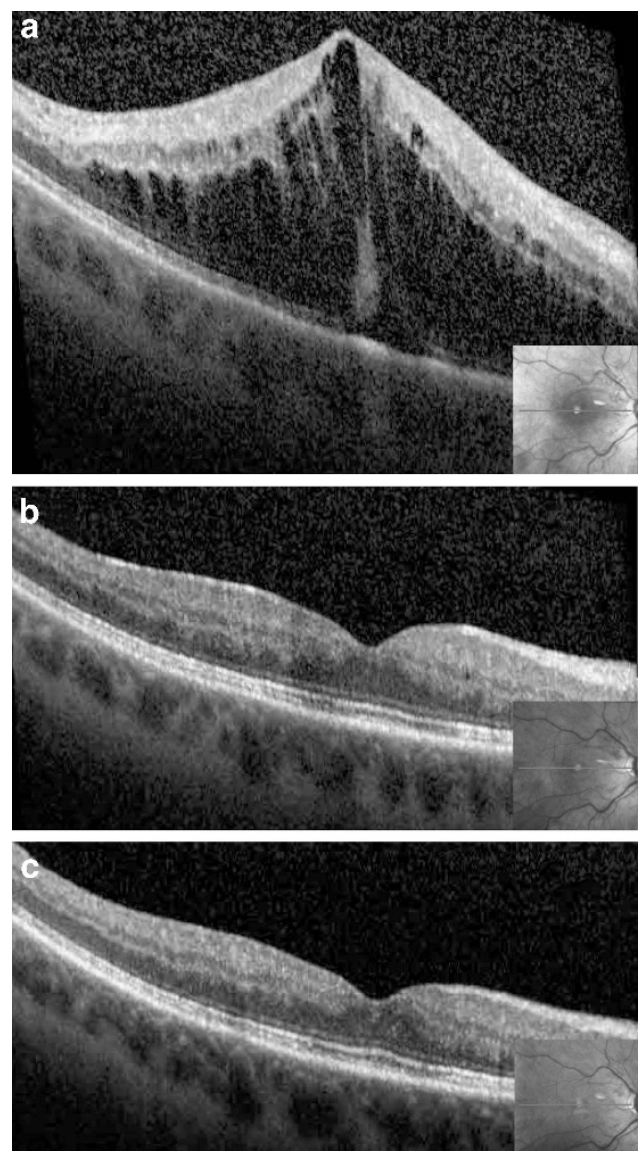

Figure 4 A 59-year-old woman presented with a CRVO with MO in her right eye. Her vision at presentation was 20/800. She received one injection of bevacizumab and two injections of ranibizumab with improvement in her vision to 20/80 after 3 months. Her CRT had decreased from $1108 \mu \mathrm{m}$ at presentation to $816 \mu \mathrm{m}$ at the 3-month follow-up (a). At this point, we decided to switch her to aflibercept given the significant persistent oedema. One month after the first injection of aflibercept, her vision was 20/20 and the retina was dry (b). She has received another five monthly injections of aflibercept and her vision 6 months after the switch is still $20 / 20$ and there is no MO (c).

with restoration of macular anatomy and no difference in VA were seen in patients with wet AMD who were switched from bevacizumab or ranibizumab to aflibercept for refractory oedema. ${ }^{18-20}$

Eadie $e t a l^{12}$ reported their findings on six patients with MO after CRVO who had persistent oedema after repeated injections with bevacizumab or ranibizumab.

Their patients had on average more injections preaflibercept than in our study (17.8 vs 6). Three out of the six patients had modest visual gains and had improvement in the macular anatomy. The mean followup was 7 months.

Our patients received on average six injections of either bevacizumab or ranibizumab before the switch to aflibercept. There were 14 patients who received less than six total injections before the switch. Although the time for clinically significant VA gain in the CRUISE study was 5.2 months, ${ }^{21}$ we decided to include patients in this study with a minimum of three prior injections of bevacizumab or ranibizumab based on the poor visual outcome of the early non-responders reported by Bhisitkul et al. ${ }^{8}$

The limitations of this study include the lack of standardized VA measurement, the lack of masking for the VA and OCT measurements, and the relatively small size of the study population. Its retrospective design naturally excludes a controlled environment for this cohort of patients as well as standardization of the treatment protocol. In addition, the CRT measurements are subject to automated segmentation errors as they were extracted from the retinal thickness profile maps of the two OCT platforms utilized. Although the improvement in the VA was statistically significant after one injection of aflibercept, that effect was not sustained at the end of the follow-up. A possible reason could be under-powering as a result of small sample size, as this precludes a more detailed subgroup analysis based on initial treatment, history of panretinal photocoagulation, and/or history of prior intravitreal steroid therapy.

In summary, aflibercept can lead to improvement in macular anatomy with VA stabilization in eyes with MO after CRVO that are not responsive to ranibizumab or bevacizumab. Further larger randomized trials are needed to determine the efficacy of aflibercept in this subset of patients with CRVO.

\section{Summary}

What was known before

- Bevacizumab or ranibizumab can decrease macular oedema in patients with central retinal vein occlusion (CRVO).

- Aflibercept is the latest agent used in clinical practice for patients with macular oedema due to CRVO.

- Some patients are not responsive or develop tachyphylaxis to bevacizumab or ranibizumab.

What this study adds

- We evaluated the efficacy of aflibercept in patients with macular oedema secondary to CRVO resistant to bevacizumab or ranibizumab.

- This resulted in stabilization of the vision, improved macular anatomy, and extension of the injection interval.

\section{Conflict of interest}

The authors declare no conflict of interest.

\section{References}

1 Evaluation of grid pattern photocoagulation for macular edema in central vein occlusion. The Central Vein 
Occlusion Study Group M report. Ophthalmology 1995; 102: 1425-1433.

2 Ip MS, Scott IU, VanVeldhuisen PC, Oden NL, Blodi BA, Fisher $\mathrm{M}$ et al. A randomized trial comparing the efficacy and safety of intravitreal triamcinolone with observation to treat vision loss associated with macular edema secondary to central retinal vein occlusion: the Standard Care vs Corticosteroid for Retinal Vein Occlusion (SCORE) Study Report 5. Arch Ophthalmol 2009; 127: 1101.

3 Haller JA, Bandello F, Belfort R, Blumenkranz MS, Gillies M, Heier J et al. Dexamethasone intravitreal implant in patients with macular edema related to branch or central retinal vein occlusion twelve-month study results. Ophthalmology 2011; 118: 2453-2460.

4 Brown DM, Campochiaro PA, Singh RP, Li Z, Gray S, Saroj $\mathrm{N}$ et al. Ranibizumab for macular edema following central retinal vein occlusion: six-month primary end point results of a phase III study. Ophthalmology 2010; 117: 1124-1133.e1.

5 Prager F, Michels S, Kriechbaum K, Georgopoulos M, Funk M, Geitzenauer W et al. Intravitreal bevacizumab (Avastin) for macular oedema secondary to retinal vein occlusion: 12-month results of a prospective clinical trial. Br J Ophthalmol 2009; 93: 452-456.

6 Zhang H, Liu Z-L, Sun P, Gu F. Intravitreal bevacizumab for treatment of macular edema secondary to central retinal vein occlusion: eighteen-month results of a prospective trial. J Ocul Pharmacol Ther 2011; 27: 615-621.

7 Campochiaro PA, Brown DM, Awh CC, Lee SY, Gray S, Saroj $\mathrm{N}$ et al. Sustained benefits from ranibizumab for macular edema following central retinal vein occlusion: twelve-month outcomes of a phase III study. Ophthalmology 2011; 118: 2041-2049.

8 Bhisitkul RB, Campochiaro PA, Shapiro H, Rubio RG. Predictive value in retinal vein occlusions of early vs late or incomplete ranibizumab response defined by optical coherence tomography. Ophthalmology 2013; 120: 1057-1063.

9 Papadopoulos N, Martin J, Ruan Q, Rafique A, Rosconi MP, Shi E et al. Binding and neutralization of vascular endothelial growth factor (VEGF) and related ligands by VEGF Trap, ranibizumab and bevacizumab. Angiogenesis 2012; 15: 171-185.

10 Korobelnik J-F, Holz FG, Roider J, Ogura Y, Simader C, Schmidt-Erfurth $U$ et al. Intravitreal aflibercept injection for macular edema resulting from central retinal vein occlusion: one-year results of the Phase 3 GALILEO Study. Ophthalmology 2014; 121: 202-208.

11 Heier JS, Clark WL, Boyer DS, Brown DM, Vitti R, Berliner AJ et al. Intravitreal aflibercept injection for macular edema due to central retinal vein occlusion: two-year results from the COPERNICUS study. Ophthalmology 2014; 121: 1414-1420.e1.

12 Eadie JA, Ip MS, Kulkarni AD. Response to aflibercept as secondary therapy in patients with persistent retinal edema due to central retinal vein occlusion initially treated with bevacizumab or ranibizumab. Retina (Philadelphia, Pa) 2014; 34: 2439-2443.

13 Boyer D, Heier J, Brown DM, Clark WL, Vitti R, Berliner AJ et al. Vascular endothelial growth factor Trap-Eye for macular edema secondary to central retinal vein occlusion: six-month results of the phase 3 COPERNICUS study. Ophthalmology 2012; 119: 1024-1032.

14 Holz FG, Roider J, Ogura Y, Korobelnik JF, Simader C, Groetzbach G et al. VEGF Trap-Eye for macular oedema secondary to central retinal vein occlusion: 6-month results of the phase III GALILEO study. Br J Ophthalmol 2013; 97: 278-284.

15 Brown DM, Heier JS, Clark WL, Boyer DS, Vitti R, Berliner AJ et al. Intravitreal aflibercept injection for macular edema secondary to central retinal vein occlusion: 1-year results from the phase 3 COPERNICUS study. Am J Ophthalmol 2013; 155: 429-437.e7.

16 Ogura Y, Roider J, Korobelnik J-F, Holz FG, Simader C, Schmidt-Erfurth U et al. Intravitreal aflibercept for macular edema secondary to central retinal vein occlusion: 18-month results of the Phase 3 GALILEO Study. Am J Ophthalmol 2014; 158: 1032-1038.e2.

17 Saint-Geniez M, Maharaj AS, Walshe TE, Tucker BA, Sekiyama E, Kurihara T et al. Endogenous VEGF is required for visual function: evidence for a survival role on müller cells and photoreceptors. PLOS ONE 2008; 3: e3554.

18 Yonekawa Y, Andreoli C, Miller JB, Loewenstein JI, Sobrin L, Eliott $\mathrm{D}$ et al. Conversion to aflibercept for chronic refractory or recurrent neovascular age-related macular degeneration. Am J Ophthalmol 2013; 156: 29-35.e2.

19 Bakall B, Folk JC, Boldt HC, Sohn EH, Stone EM, Russell SR et al. Aflibercept therapy for exudative age-related macular degeneration resistant to bevacizumab and ranibizumab. Am J Ophthalmol 2013; 156: 15-22.e1.

20 Ho VY, Yeh S, Olsen TW, Bergstrom CS, Yan J, Cribbs BE et al. Short-term outcomes of aflibercept for neovascular age-related macular degeneration in eyes previously treated with other vascular endothelial growth factor inhibitors. Am J Ophthalmol 2013; 156: 23-28.e2.

21 Thach AB, Yau L, Hoang C, Tuomi L. Time to clinically significant visual acuity gains after ranibizumab treatment for retinal vein occlusion: BRAVO and CRUISE trials. Ophthalmology 2014; 121: 1059-1066. 